\title{
IMPROVED TOPOGRAPHIC MAPPING IN VEGETATED MOUNTAINOUS AREAS BY HIGH-RESOLUTION RADARGRAMMETRY-ASSISTED SAR INTERFEROMETRY
}

\author{
Yuting Dong ${ }^{1,2}$, Lu Zhang ${ }^{3, *}$, Mingsheng Liao ${ }^{3}$ \\ ${ }^{1}$ School of Geography and Information Engineering, China University of Geosciences, Wuhan, China - dongyt@cug.edu.cn \\ ${ }^{2}$ Remote Sensing Technology Institute (IMF), German Aerospace Center (DLR), Oberpfaffenhofen, Germany \\ ${ }^{3}$ State Key Laboratory of Information Engineering in Surveying, Mapping and Remote Sensing (LIESMARS), Wuhan University, \\ Wuhan, China - luzhang@whu.edu.cn, liao@whu.edu.cn
}

Commission III, WG III/3

KEY WORDS: Radargrammetry; SAR Interferometry; DEM; Vegetated Mountainous Areas; Terrain Mapping

\begin{abstract}
:
In densely vegetated mountainous areas, the height measurement accuracy of SAR interferometry is contaminated by phase unwrapping errors and volume scattering effects of the vegetation. Aiming at this limitation of InSAR, we propose the highresolution radargrammetry-assisted SAR interferometry method to achieve high-precision topographic mapping. Specifically speaking, we propose to apply our methods in the TerraSAR-X images with a repeat-pass InSAR pair and a stereo radargrammetric pair sharing the same master image which can avoid radarcoding the reference DEM and minimize the acquisition time discrepancy between the reference DEM and InSAR data. Compared with medium-resolution reference DEM, the high-resolution radargrammetric DEM can simulate the reference topographic phase more accurately which reduces the InSAR phase unwrapping errors. In addition to the removal of the reference topographic phase, we automatically select reliable points from radargram metric DEM for calibrating the absolute InSAR phase offset. Finally, we fuse the radargrammetric DEM and InSAR DEM together with weights determined from theoretical height error to fill in data voids in InSAR DEM. The experimental results show that the DEM generated by radargrammetry-assisted SAR interferometry has better height accuracy than DEM generated by either single method.
\end{abstract}

\section{INTRODUCTION}

As one of two major approaches (SAR interferometry and radargrammetry) using SAR remote sensing data for topographic mapping (Thierry Toutin 2000), InSAR makes use of the interferometric phase information to extract the terrain height accurately, which has been successfully applied for global DEM generation by SRTM (Shuttle Radar Topography Mission) and TanDEM-X (TerraSAR-X Add-on for Digital Elevation Measurements) mission (Krieger et al., 2007). However, the height accuracy of InSAR at the vegetated area is contaminated by the volume scattering effects of vegetation. Furthermore, the InSAR phase measurement corresponds to the relative range difference between the master and slave satellites, which needs to be unwrapped and calibrated to reconstruct the absolute topographic phase. In the mountainous areas, the interferometric phase is prone to phase unwrapping errors and it lacks the natural or man-made corner reflectors for absolute topographic phase calibration. As a complementary SAR method for topographic mapping, radargrammetry makes use of the amplitude information and hence it is not affected by phase unwrapping errors and volume scattering decorrelation. Besides, radargrammetry determines the absolute ground position of homologous points through forward intersection model. Hence, radargrammetric height measurement can promisingly assist InSAR absolute topographic reconstruction.

Due to the complementary nature of InSAR and radargrammetry, researchers have tried to combine these two methods together to realize high-precision topographic mapping. In order to realize the calibration of InSAR unwrapped phase, researchers proposed the stereo interferometric SAR (StInSAR) method which takes advantage of StereoSAR to determine absolute positioning of natural or man-made reflectors and then apply these points as ground control points for InSAR unwrapped phase calibration (Eldhuset, 2017; Eldhuset and Weydahl, 2013). The prerequisite of StInSAR is the existence of the natural or manmade reflectors which limits its application in densely vegetated mountainous areas with no evident natural corner reflectors. Researchers also tried to generate the radargrammetric DEM from a stereo radargrammetric pair which is then used to flatten the interferogram, calibrate the unwrapped phase and provide height estimates for missing InSAR-DEM values (Gelautz et al., 2003). However, this procedure is influenced by spatial resolution and height accuracy of radargrammetric DEM which means the mediumresolution reference DEM cannot reduce the fringes significantly at rolling terrain and the height error of the reference DEM excessing the InSAR height ambiguity can introduce erroneous phase unwrapping. Besides, the geometric distortions and temporal change of the reference radargrammetric DEM also influence the height accuracy of the InSAR processing. In order to minimize the frequency of interferometric fringes and at the same time not to introduce additional topographic measurement errors, the reference DEM must guarantee a certain resolution and height accuracy.

With launch of the high-resolution spaceborne satellites, such as TerraSAR-X, COSMO-SkyMed and RADARSAT-2, the spatial resolution and height accuracy of the stereo radargrammetric DEM have been accordingly increased (Arief et al., 2019; Capaldo et al., 2011; de Oliveira et al., 2011; Guimarães et al., 2018; Hao et al., 2019; Toutin, 2010). Our recent stereo radargrammetric studies with Stripmap mode

\footnotetext{
* Corresponding author
} 
TerraSAR-X images have shown promising results that the root mean square error (RMSE) of radargrammetric DEM are up to $6 \mathrm{~m}$ over flat areas and up to $15 \mathrm{~m}$ over mountainous areas and the spatial resolution is about $10 \mathrm{~m}$ (Dong et al., 2018).

With the high-resolution and high-precision radargrammetric DEM, we propose the high-resolution radargrammetry-assisted SAR interferometry method for improved topographic mapping at vegetated mountainous areas. In detail, we propose to apply our methods in the TerraSAR-X images with a repeat-pass InSAR pair and a stereo radargrammetric pair. The TerraSAR$\mathrm{X}$ repeat-pass data have much more data archives and can have smallest acquisition time discrepancy with the TerraSAR-X stereo radargrammetric pair. Moreover, we make the TerraSAR-X stereo radargrammetric pair and the InSAR pair to share the same master image which means the radargrammetric DEM before geocoding has the same imaging geometry with the InSAR pair and can be directly used as reference DEM for InSAR pair and avoid the height error caused by radarcoding. In comparison with the open-source medium-resolution reference DEMs, the high-resolution radargrammetric DEM can simulate the reference topographic phase in more detail which improves the InSAR phase unwrapping. In addition to the removal of the reference topographic phase, we automatically select reliable points from radargrammetric DEM based on the theoretical height errors of SAR interferometry and radargrammetry with empirically determined thresholds. As to fill in the data voids and correct the residual phase unwrapping errors in InSAR DEM with radargrammetric DEM, we develop a fusion method with weights determined from the theoretical height errors.

In Section 2, the experimental area and datasets are introduced. The detailed methodology for high-resolution radargrammetryassisted InSAR DEM generation is described in Section 3. Section 4 shows the results of our experiments at test area Mount Song, China. In Section 5, the final conclusions are drawn.

\section{STUDY AREA AND DATASETS}

\subsection{Study area}

The study area is located at Mount Song area in Henan province of central China, as shown in Figure 1. This area is selected as study area because the terrain is complex and variable. The elevation ranges from $150 \mathrm{~m}$ to $1500 \mathrm{~m}$ above sea level, and the slopes in the central part are very steep. The study area is densely vegetated, causing a severe decorrelation problem for InSAR analysis, thus stereo radargrammetry can be a good complimentary technique when mapping the topography of this area.

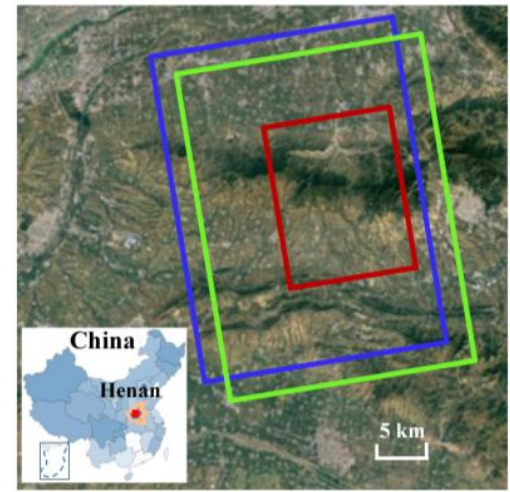

Figure 1. Coverage of study area and datasets used. The blue and green rectangles mark the coverage of TerraSAR-X images acquired in 12.07.2011 and 18.07/29.07.2011 respectively; the red rectangle marks the coverage of the photogrammetric DEM.

\subsection{TerraSAR-X datasets}

The SAR data used in this experiment are three TerraSAR-X single-look complex (SLC) images in the Stripmap mode. Their detailed parameters are listed in Table 1. Two of them share the same incident angle (ID: 0718 and ID: 0729) and can form an interferometric pair and two of them with different incident angle (ID: 0718 and ID: 0712) form a stereo pair. The interferometric pair and stereo pair share the same master image (ID: 0718). For the repeat-pass interferometry of TerraSAR-X data (ID: 0718 and ID: 0729), the shortest revisit time is $11 \mathrm{~d}$. The Normal baseline is $251 \mathrm{~m}$ and the corresponding height ambiguity is $22 \mathrm{~m}$, which is a good compromise between the geometric decorrelation and height measurement accuracy.

\begin{tabular}{|c|c|c|c|c|}
\hline ID & $\begin{array}{c}\text { Acquisition } \\
\text { Date }\end{array}$ & $\begin{array}{c}\text { Orbit } \\
\text { Direction }\end{array}$ & $\begin{array}{c}\text { Incidence } \\
\text { Angle }\left(^{\circ}\right)\end{array}$ & $\begin{array}{c}\text { Resolution } \\
\text { rg/az* (m) }\end{array}$ \\
\hline 0712 & 12.07 .2011 & Ascending & 44.5 & $1.8 / 3.3$ \\
0718 & 18.07 .2011 & Ascending & 28.9 & $1.2 / 3.3$ \\
0729 & 29.07 .2011 & Ascending & 28.9 & $1.2 / 3.3$ \\
\hline
\end{tabular}

Table 1. Parameters of the experimental data

\subsection{Photogrammetric DEM}

In order to evaluate the height accuracy of the generated DEMs, a photogrammetric DEM with $1 \mathrm{~m}$ resolution is used as the ground truth. The height accuracy of the photogrammetric DEM is better than $1 \mathrm{~m}$. The photogrammetric DEM was acquired in 2011 which is close to the acquisition time of TerraSAR-X images used. The coverage of the photogrammetric DEM is marked by the red rectangle in Figure 1 inside the coverage of the TerraSAR-X images marked by the blue and green rectangles in Figure 1. There are different terrain types, such as mountains and plains, inside the coverage of the photogrammetric DEM, making it suitable for height accuracy evaluation of the generated DEMs in different kinds of terrain conditions.

\section{METHODOLOGY}

\subsection{InSAR reference topographic phase removal by radargrammetric DEM}

In order to ease the difficulty of phase unwrapping, the external DEM is used to simulate the reference topographic phase and then removed from the interferogram. Thus the frequency of fringes is greatly reduced and the accuracy of phase unwrapping is improved. The open-source DEMs such as, SRTM DEM $30 \mathrm{~m}$, ASTER GDEM $30 \mathrm{~m}$, AW3D30 30m or TanDEM-X global DEM $90 \mathrm{~m}$ are too coarse for the highresolution SAR data at about $1-5 \mathrm{~m}$ resolution. Besides there is often acquisition time discrepancy between the SAR images and open-source reference DEMs which is likely to introduce height errors into phase unwrapping and absolute phase calibration due to temporal terrain changes. 
Therefore the radargrammetric DEM with similar spatial resolution with the InSAR DEM is used to substitute the opensource DEMs as the reference DEM. For the radargrammetric pair which shares the same master image with the InSAR pair, the radargrammetric DEM is generated in the same image space as the unwrapped phase and thus save the radarcoding step. It is worth mentioning that the height error of the reference DEM should not be more than the height ambiguity of the InSAR pair or it will introduce full-cycle phase jumps and introduce extra phase unwrapping errors.

\subsection{InSAR absolute topographic phase calibration with radargrammetric DEM}

After removal of the flat terrain phase and the reference topographic phase, the differential interferometric phase can be comprised of four components as in Equation 1.

$$
\phi_{\text {diff }}=\phi_{\text {topo_residual }}+\phi_{\text {def }}+\phi_{\text {atm }}+\phi_{\text {noise }}
$$

where $\quad \phi_{\text {diff }}=$ differential interferometric phase

$\phi_{\text {topo_residual }}=$ residual topographic phase

$\phi_{\text {def }}=$ ground deformation phase

$\phi_{\text {atm }}=$ Atmospheric Phase Screen (APS)

$\phi_{\text {noise }}=$ phase noise

$\phi_{\text {topo_residual }}$ corresponds to the height difference between topographic height and reference height. $\phi_{\text {def }}$ can be generally ignored by using a short temporal baseline of a few days. For the repeat-pass TerraSAR-X InSAR images, $\phi_{a t m}$ would introduce significant errors in the DEM and must be compensated. The APS consists of a vertically stratified component and a turbulent mixing one (Hanssen, 2001). Based on spatial pattern analyses of these two APS components, an elevation-to-phase regression model and an adaptive low-pass filter are employed to estimate and remove them from the differential interferogram sequentially (Liao et al., 2013). $\phi_{\text {noise }}$ is caused by the thermal noise of the SAR sensor as well as the decorrelation between two SAR acquisitions, and its impact can be mitigated through interferogram filtering.

After the APS removal, the residual phase signal in the differential interferogram is reasonably assumed to represent local topographic details that is not be portrayed by the reference DEM. The differential interferogram is unwrapped by using the established statistical cost network flow minimization (MCF) algorithm (Chen and Zebker, 2001) and the unwrapped differential phase is $\phi_{u n w}$. There is always offset $\phi_{\text {off }}$ between the unwrapped differential phase $\phi_{u n w}$ and absolute differential phase $\left(\phi_{\text {topo }}-\phi_{\text {ref }}\right)$, as shown in Figure 2, and therefore phase calibration is needed to determine this offset.

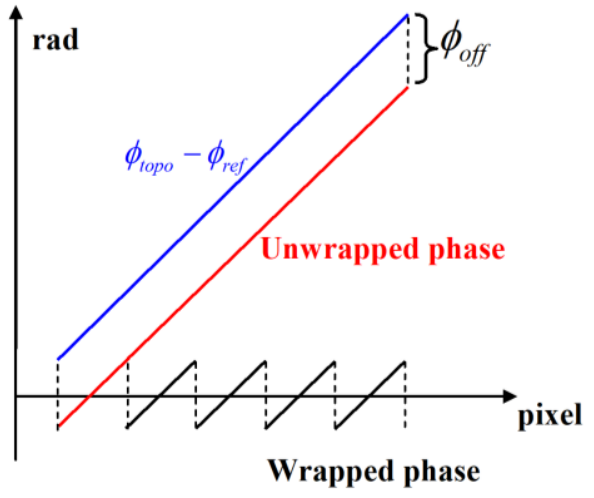

Figure 2 Absolute phase offset calibration

In order to determine the absolute phase offset, we select reliable points with absolute height measurement from the reference radargrammetric DEM. The candidate points should also have high height measurement accuracy in the InSAR DEM. Hence, the automatic selection criterion relies on the standard deviation of height errors of InSAR DEM $\sigma_{h, I n S A R}$ and radargrammetric DEM $\sigma_{h, \text { stereo }}$. The threshold for selection is set empirically. In our experiments, we set the threshold as $\sigma_{h, I n S A R}$ no more than $2 \mathrm{~m}$ and $\sigma_{h, \text { stereo }}$ no more than $1 \mathrm{~m}$. The phase offset at each sample points $\phi_{\text {off }, i}$ can be written as Equation 2.

$$
\phi_{o f f, i}=-\phi_{u n w, i}
$$

where $\quad \phi_{u n w, i}=$ absolute phase offset at sample point $i$

$$
\phi_{u n w, i}=\text { unwrapped differential phase at point } i
$$

We plot the distribution frequency histogram of $\phi_{\text {off }, i}$. The mean value over the main lobe of the histogram provides the absolute phase offset $\phi_{\text {off }}$. The side lobe of this plotted histogram reveals the existence of the phase unwrapping errors which can be checked and corrected.

\subsection{Derivation of theoretical height error and DEM fusion weights}

The absolute topographic phase $\phi_{\text {topo }}$ can be converted into terrain height $h_{I n S A R}$ with the InSAR height ambiguity $H_{a m b}$ which is the height value corresponding to one cycle phase. Similarly, there is linear conversion relationship between the disparity value $\Delta p$ and the stereo height $h_{\text {Stereo }}$ as in Table 2 where $\delta_{R}$ and $\theta_{2}$ are the sampling space in range direction and incidence angle of the slave image of the stereo pair. Therefore, the standard deviations of height errors of SAR interferometry $\sigma_{h, I n S A R}$ and radargrammetry $\sigma_{h, \text { stereo }}$ can be estimated from the standard deviations of phase noise $\sigma_{\phi}$ and stereo image matching error $\sigma_{p}$ based on error propagation law in Table 2. 


\begin{tabular}{|l|l|}
\hline \multicolumn{1}{|c|}{ Stereo Radargrammetry } & \multicolumn{1}{c|}{ SAR Interferometry } \\
\hline Theoretical disparity error $\sigma_{p}$. & Theoretical phase error $\sigma_{\phi}$. \\
\hline $\begin{array}{l}\text { Linear relationship between } \\
\text { disparity and height: }\end{array}$ & $\begin{array}{l}\text { Linear relationship between } \\
\text { phase and height: } \\
h_{\text {Stereo }}=\frac{\delta_{R}}{\cos \theta_{2}} \Delta p .\end{array}$ \\
$\begin{array}{l}\text { Theoretical height error: } \\
h_{\text {InSAR }}=\frac{H_{\text {amb }}}{2 \pi} \phi_{\text {topo }} .\end{array}$ & Theoretical height error: \\
$\sigma_{h, \text { stereo }}=\frac{\delta_{R}}{\cos \theta_{2}} \sigma_{p}$ & $\sigma_{h, I n S A R}=\frac{H_{\text {amb }}}{2 \pi} \sigma_{\phi}$ \\
\hline
\end{tabular}

Table 2. Theoretical height errors derivation process of radargrammetric and InSAR height

In order to determine the fusion weights of InSAR DEM and radargrammetric DEM, we take advantage of the sigmoid function to convert the standard deviation of height errors in Table 2 into weights between $[0,1]$. Equation 3 is the $\mathrm{x}$-axis symmetric sigmoid function intercepted between $[-10,10]$ and Figure 3 shows the corresponding plot of Equation 3. Two feature points are marked in Figure 3, which are $w(-3)=0.95$ and $w(3)=0.05$.

$$
w(x)=\frac{1}{1+e^{x}}, x \in[-10,10]
$$

where $\quad x=$ independent variable

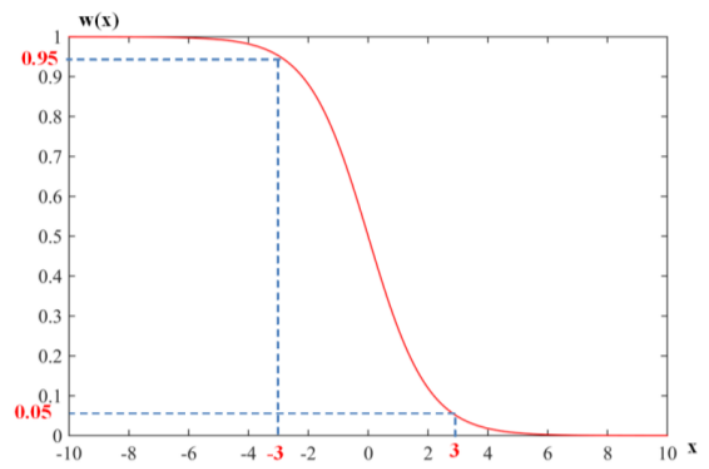

Figure 3 Plot of the $\mathrm{x}$-axis symmetric sigmoid function corresponding to Equation 3

In order to determine the fusion weights, the 5\% and $95 \%$ quantiles are calculated from the standard deviations of height errors (both $\sigma_{h, I n S A R}$ and $\sigma_{h, \text { stereo }}$ ), which are $\sigma_{h, 5 \%}$ and $\sigma_{h, 95 \%} \cdot \sigma_{h}$ varying between $\sigma_{h, 5 \%}$ and $\sigma_{h, 95 \%}$ is scaled to [3,3] and then use Equation 3 to calculate its relative weight for elevation fusion. $\sigma_{h}$ larger than $\sigma_{h, 95 \%}$ is thought to be untrustworthy and set 0 as fusion weight, while $\sigma_{h}$ smaller than $\sigma_{h, 5 \%}$ is viewed as correct value and set 1 as fusion weight. With the determined weights, then the fused height is calculated based on Equation 4.

$$
h_{\text {fusion }}=\frac{h_{\text {stereo }} \cdot w_{\text {stereo }}+h_{\text {InSAR }} \cdot w_{\text {InSAR }}}{w_{\text {stereo }}+w_{\text {InSAR }}}
$$

$$
\begin{array}{ll}
\text { where } \quad & h_{\text {fusion }}=\text { fused height } \\
& h_{\text {stereo }}=\text { stereo radargrammetric height }
\end{array}
$$

$$
\begin{aligned}
& w_{\text {stereo }}=\text { weight for radargrammetric height } \\
& h_{\text {insar }}=\text { InSAR height } \\
& w_{\text {InSAR }}=\text { weight for InSAR height }
\end{aligned}
$$

\section{EXPERIMENTS}

\subsection{Experimental results}

There is $11 \mathrm{~d}$ interval between the InSAR pair and the atmospheric phase caused non-negligible effect in the interferometric phase. Therefore the combined filter proposed by Liao et al, 2013 was used to remove the atmospheric phase. The simulated atomospheric phase is shown in Figure 4 (b) and the differential interferogram before and after atmospheric phase removal are presented in Figure 4 (a) and 4 (c) respectively and we can see from Figure 4 that most of the lowfrequency atmospheric phase was filtered and the highfrequency residual topographic phase remained.

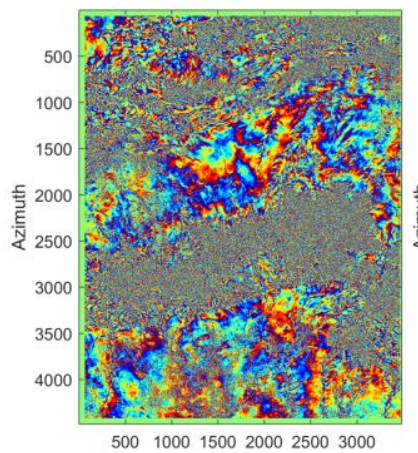

(a)

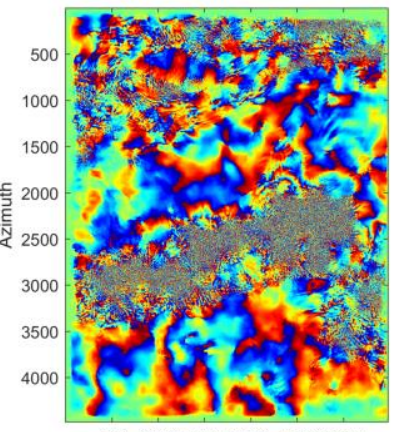

50010001500200025003000

(b)

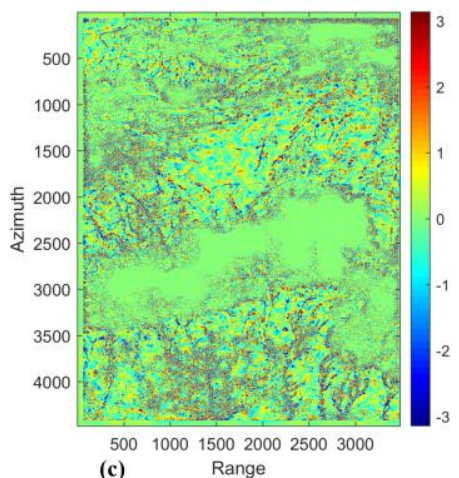

(c)

Figure 4 Atmospheric phase screen removal. (a) Re-flattened differential interferogram with rough topographic phase removed. (b) Simulated atmospheric phase based on Liao et al, 2013. (c) Re-flattened differential interferogram with the simulated atmospheric phase removed.

In order to realize automatically absolute InSAR phase calibration, about 840,000 points were selected with the height error of radargrammetric DEM less than $1 \mathrm{~m}$ and InSAR DEM less than $2 \mathrm{~m}$. Phase offsets at these selected sample points were calculated and the frequency histogram was plotted as in Figure 5. The mean value of these phase offsets was the phase offset to be calibrated. The absolute topographic phase calibration with radargrammetric DEM had another advantage 
that it can help eliminate the systematic height offset between the InSAR height and radargrammetric height.

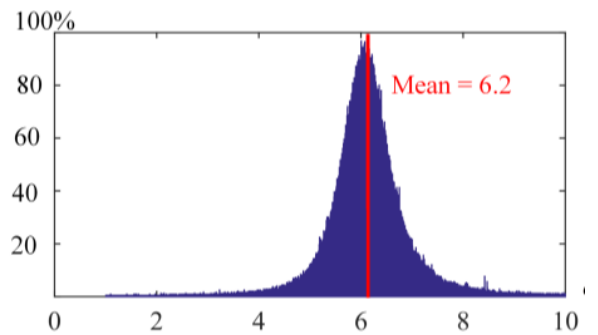

Figure 5 Absolute topographic phase offset calibration

For the interferometric pair, the pixels with the coherence less than 0.2 were masked out and eliminated from the phase unwrapping process. Hence there were lots of voids in the generated InSAR DEM covering almost all the mountainous areas with dense vegetation. The radargrammetric DEM with theoretical height error less than $7 \mathrm{~m}$ remained. Then the radargrammetric DEM was fused with InSAR DEM based on Equation 4 and this step was implemented in the SAR image space as in Figure 6. Since the interferometric pair and the stereo pair shared the same master image, there was no need for the DEMs co-registration step. The fused DEM was shown in Figure 6 (c). The percentages of valid cells of the InSAR DEM Figure 6 (a) and radargrammetric DEM Figure 6 (b) were $74.2 \%$ and $99.06 \%$, respectively, while the percentage of valid cells of the fused DEM (Figure 6 (c)) was $99.77 \%$.
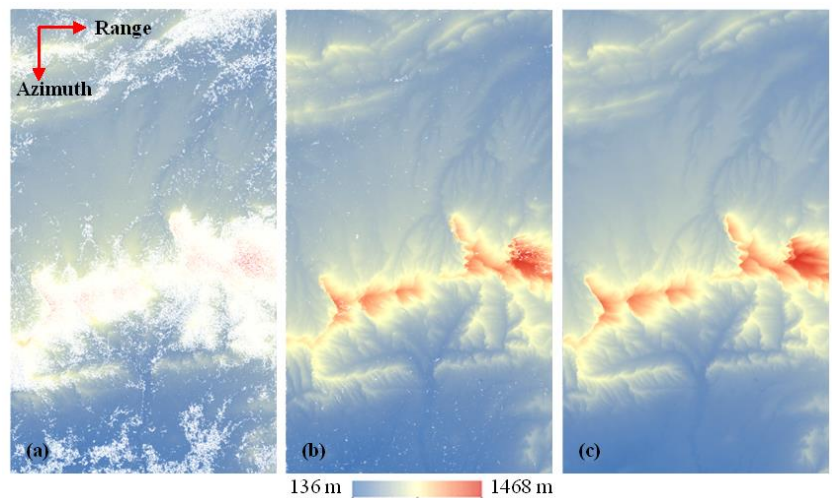

Figure 6. The InSAR DEM and radargrammetric DEM fusion process in SAR image space. (a) the InSAR DEM; (b) the radargrammetric DEM; (c) the fused DEM.

\subsection{Accuracy assessment of the resultant DEMs}

To evaluate the quality of DEMs quantitatively, we employed the photogrammetric DEM with spatial resolution of $1 \mathrm{~m}$ as the reference elevation dataset. The InSAR DEM, radargrammetric DEM and fused DEM were geocoded to the geodetic coordinate system and the height datum was the WGS84 ellipsoid. The reference DEM was transformed into the same coordinate system and height datum with the geocoded DEMs. Taking into account the difference in spatial resolution ( $1 \mathrm{~m}$ vs. $10 \mathrm{~m})$ between the reference DEM and the three geocoded DEMs, we used the nearest-neighbour resampling approach to downgrade the resolution of reference DEM to $10 \mathrm{~m}$. Afterwards, differencing between each geocoded DEM and the reference DEM was performed on the cell-to-cell basis.
In order to show the DEM details, we select two sample areas which are one plain area and one mountain area corresponding to the blue and red rectangles in Figure 7 (c), respectively. Figure 8 and 9 show the zoom-in hillshade maps of different DEMs at the two sample areas. By comparing the InSAR DEMs in Figure 8 and 9 (a) with the radargrammetric DEMs in Figure 8 and 9 (b), it can be seen that the InSAR DEMs contains more detailed terrain information while it have more date voids than the radargrammetric DEMs. Especially the InSAR DEM in Figure 8 (a) can clearly reflect a road in the middle which does not exist in the radargrammetric DEM in Figure 8 (b). The fused DEMs in Figure 8 and 9 (c) reserve the meaningful terrain details from the InSAR DEMs and meanwhile fill in the data voids in InSAR DEMs with the radargrammetric DEMs. The similarity between the fused DEMs and the photogrammetric DEMs in Figure 8 and 9 (d) validates the effectiveness of the fusion method qualitatively. The statistics of height errors of the radargrammetric DEM and fused DEM were shown in Table 3 and the height difference values as cumulative percentages were shown in Table 4. In Table 3, the mean height error, Root Mean Square Error (RMSE) and 90\% quantile of height error were calculated. Since there are much data voids in the InSAR DEM, it is not fair to compare the statistics with different sample points and thus the height errors statistics of InSAR DEM are not listed here. In Table 3, the fused DEM has smaller RMSEs and 90\% LEs than the radargrammetric DEM at the two sample areas and whole validation area. In Table 4 , the fused DEM has larger cumulative percentage of height difference than that of the radargrammetric DEM at each interval. Therefore, it can be seen that the fused DEM had higher height accuracy than the radargrammetric DEM and fewer data voids than InSAR DEM for both plains and mountains.

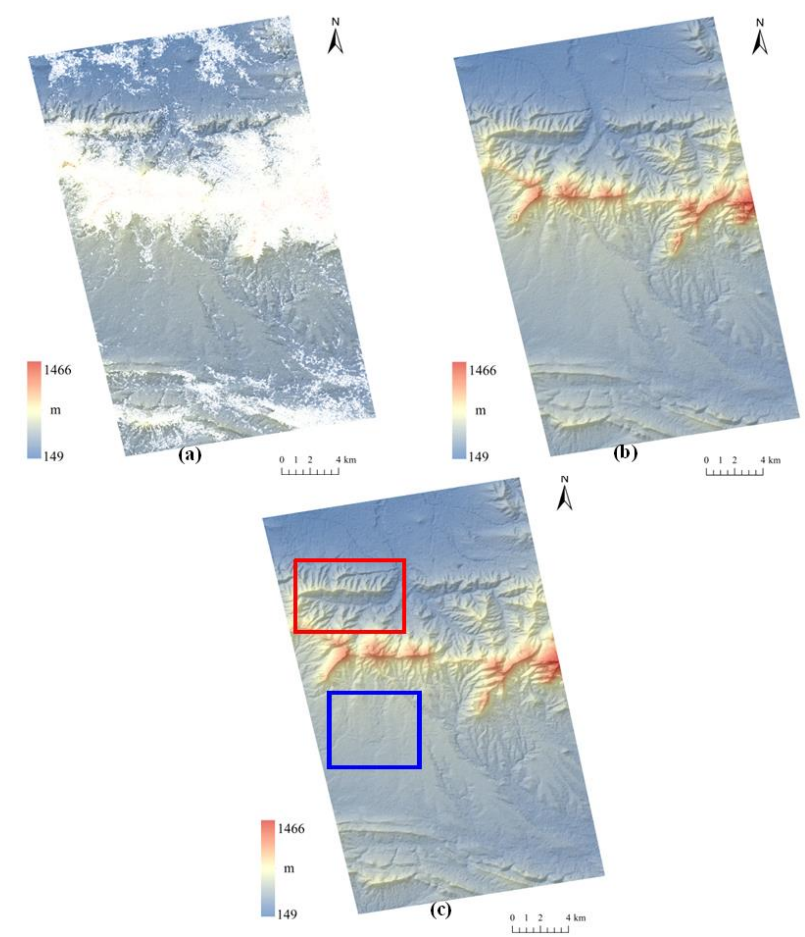

Figure 7. Hillshade maps of the geocoded DEMs. (a) the InSAR DEM; (b) the radargremmetric DEM; (c) the fused DEM. Voids are shown in white. The blue and red rectangles mark the plain and mountain sample areas. 

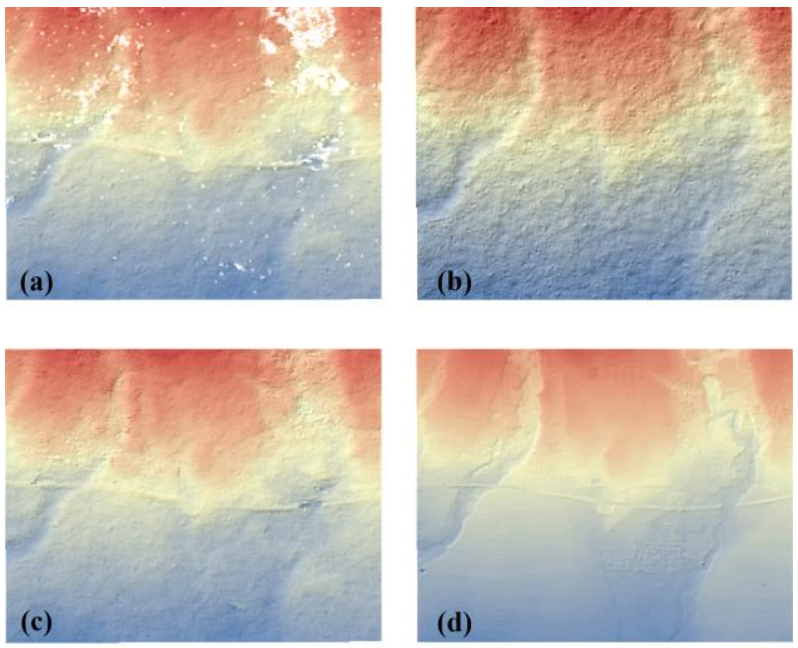

$450 \mathrm{~m}$ $569 \mathrm{~m}$

Figure 8. DEM hillshade map of the plain sample area. (a) InSAR DEM; (b) radargrammetric DEM; (c) fused DEM; (d) photogrammetric DEM.
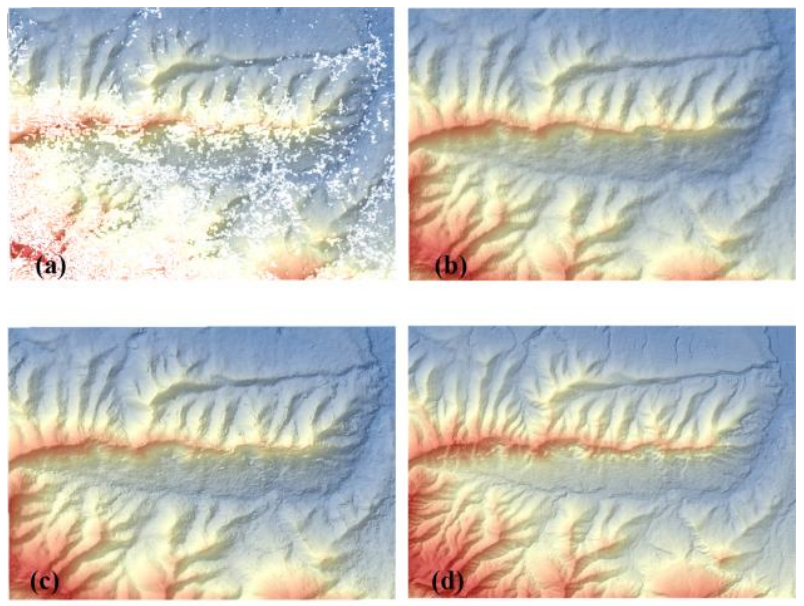

$230 \mathrm{~m}$

$940 \mathrm{~m}$

Figure 9. DEM hillshade map of the mountain sample area. (a) InSAR DEM; (b) radargrammetric DEM; (c) fused DEM; (d) photogrammetric DEM.

\begin{tabular}{|c|ccc|ccc|}
\hline & \multicolumn{3}{|c|}{ Radargrammetric DEM } & \multicolumn{3}{c|}{ Fused DEM } \\
\cline { 2 - 7 } & mea & RMS & \multirow{2}{*}{ 90\%LE } & mea & RMS & 90\%LE \\
& $\mathrm{n}$ & $\mathrm{E}$ & & $\mathrm{n}$ & \\
\hline All & 2.5 & 7.3 & 12.6 & 2.3 & 6.9 & 11.9 \\
Plain & 1.7 & 1.9 & 3.3 & 1.1 & 1.8 & 3.2 \\
$\begin{array}{c}\text { Mountai } \\
\text { n }\end{array}$ & 1.7 & 10.3 & 16.8 & 0.9 & 9.4 & 16.1 \\
\hline
\end{tabular}

Table 3 Statistical values of the height difference values (Unit: m)

\begin{tabular}{|c|cccc|}
\hline & $\leq \pm 1.0$ & $\leq \pm 5.0$ & $\leq \pm 10.0$ & $\leq \pm 20.0$ \\
\hline $\begin{array}{c}\text { Radargrammetri } \\
\text { c }\end{array}$ & 16.0 & 60.0 & 90.1 & 99.1 \\
$\begin{array}{c}\text { DEM } \\
\text { Fused DEM }\end{array}$ & 16.7 & 63.4 & 92.7 & 99.3 \\
\hline
\end{tabular}

Table 4 Height difference values as cumulative percentages (\%)

\section{CONCLUSIONS}

We propose the high-resolution radargrammetry-assisted SAR interferometry to generate DEM at vegetated mountainous areas. The proposed method is applied on Stripmap mode TerraSAR-X images with an InSAR pair and a radargrammetric pair sharing the same master image. This novel data combination method avoids the radarcoding the radargrammetric DEM into the image space of InSAR master image and with the calibration process, the systematic offset between the InSAR DEM and radargrammetric has been removed that the radargrammetic DEM and InSARDEM can be fused directly at the image space. It is preferable that the InSAR and radargrammetric data are acquired in the same season to avoid temporal changes at the terrain surface.

With the radargrammetric DEM, the absolute phase offset of the unwrapped phase is automatically calibrated, which is helpful for InSAR DEM generation at complex mountainous areas with great difficulty to collect ground control points or even point-like targets.

The radargrammetric DEM is generated from the amplitude disparity information and not affected by the volume scattering decorrelation. Hence, the radargrammetric DEM is complementary to InSAR DEM at the vegetated areas. With the fusion method proposed, the fused DEM has well maintained the terrain details and fill in data voids of the InSAR DEM. By validating the generated DEMs with the photogrammetric DEM, the fused DEM has better height accuracy than DEM generated by either single method proving that the proposed method is effective and feasible in in highprecision terrain mapping of vegetated mountainous areas.

\section{ACKNOWLEDGEMENTS}

This work was supported by the National Natural Science Foundation of China (Grant No. 41901413) and the Open Research Fund of State Key Laboratory of Information Engineering in Surveying, Mapping and Remote Sensing, Wuhan University (Grant No. 19R01).

\section{REFERENCES}

Arief, R., Setiyoko, A., Maulana, R., Ali, S., Sudiana, D., Arymurthy, A.M., 2019. Quality assessment of dem generated from sar radargrammetry based on cross-correlation and spatial resolution setting. Journal of Physics: Conference Series 1201, 012027.

Capaldo, P., Crespi, M., Fratarcangeli, F., Nascetti, A., Pieralice, F., 2011. High-resolution sar radargrammetry: A first application with cosmo-skymed spotlight imagery. IEEE Geoscience and Remote Sensing Letters 8 (6), 1100-1104.

de Oliveira, C.G., Paradella, W.R., da Silva, A.d.Q., 2011. Assessment of radargrammetric dsms from terrasar-x stripmap images in a mountainous relief area of the amazon region. ISPRS Journal of Photogrammetry and Remote Sensing 66 (1), 67-72. 
Chen, C.W., Zebker, H.A., 2001. Two-dimensional phase unwrapping with use of statistical models for cost functions in nonlinear optimization. JOSA A 18 (2), 338-351.

Dong, Y., Zhang, L., Balz, T., Luo, H., Liao, M., 2018. Radargrammetric dsm generation in mountainous areas through adaptive-window least squares matching constrained by enhanced epipolar geometry. ISPRS Journal of Photogrammetry and Remote Sensing 137, 61-72.

Eldhuset, K., 2017. Combination of stereo sar and insar for dem generation using tandem-x spotlight data. International Journal of Remote Sensing 38 (15), 4362-4378.

Eldhuset, K., Weydahl, D.J., 2013. Using stereo sar and insar by combining the cosmo-skymed and the tandem-x mission satellites for estimation of absolute height. International Journal of Remote Sensing 34 (23), 8463-8474.

Gelautz, M., Paillou, P., Chen, C., Zebker, H., 2003. Radar stereo-and interferometry-derived digital elevation models: Comparison and combination using radarsat and ers-2 imagery. International Journal of Remote Sensing 24 (24), 5243-5264.

Guimarães, U.S., da Silva Narvaes, I., Galo, M.d.L.B.T., da Silva, A.d.Q., de Oliveira Camargo, P., 2018. Radargrammetric approaches to the flat relief of the amazon coast using cosmo-skymed and terrasar-x datasets. ISPRS Journal of Photogrammetry and Remote Sensing 145, 284-296. Hao, X., Zhang, H., Wang, Y., Wang, J., 2019. A framework for high-precision dem reconstruction based on the radargrammetry technique. Remote Sensing Letters 10 (11), 1123-1131.

Krieger, G., Moreira, A., Fiedler, H., Hajnsek, I., Werner, M., Younis, M., Zink, M., 2007. Tandem-x: A satellite formation for high-resolution sar interferometry. IEEE Transactions on Geoscience and Remote Sensing 45 (11), 3317-3341.

Liao, M., Jiang, H., Wang, Y., Wang, T., Zhang, L., 2013. Improved topographic mapping through high-resolution sar interferometry with atmospheric effect removal. ISPRS Journal of Photogrammetry and Remote Sensing 80, 72-79.

Thierry Toutin , L.G., 2000. State-of-the-art of elevation extraction from satellite sar data. ISPRS Journal of Photogrammetry \& Remote Sensing 55, 13-33.

Toutin, T., 2010. Impact of radarsat-2 sar ultrafine-mode parameters on stereo-radargrammetric dems. IEEE Transactions on Geoscience and Remote Sensing 48 (10), 3816-3823. 